\title{
Methylated-UHRF1 and PARP1 interaction is critical for homologous recombination
}

\author{
Ja Young Hahm", Joo-Young Kang , Jin Woo Park, Hyeonsoo Jung E Sang-Beom Seo * \\ Department of Life Science, College of Natural Sciences, Chung-Ang University, Seoul 06974, Korea
}

\begin{abstract}
A recent study suggested that methylation of ubiquitin-like with PHD and RING finger domain 1 (UHRF1) is regulated by SET7 and lysine-specific histone demethylase 1A (LSD1) and is essential for homologous recombination (HR). The study demonstrated that SET7-mediated methylation of UHRF1 promotes polyubiquitination of proliferating cell nuclear antigen (PCNA), inducing HR. However, studies on mediators that interact with and recruit UHRF1 to damaged lesions are needed to elucidate the mechanism of UHRF1 methylationinduced HR. Here, we identified that poly [ADP-ribose] polymerase 1 (PARP1) interacts with damage-induced methylated UHRF1 specifically and mediates UHRF1 to induce HR progression. Furthermore, cooperation of UHRF1-PARP1 is essential for cell viability, suggesting the importance of the interaction of UHRF1-PARP1 for damage tolerance in response to damage. Our data revealed that PARP1 mediates the HR mechanism, which is regulated by UHRF1 methylation. The data also indicated the significant role of PARP1 as a mediator of UHRF1 methylation-correlated HR pathway. [BMB Reports 2020; 53(2): 112-117]
\end{abstract}

\section{INTRODUCTION}

DNA damage repair processes play a key role in maintaining genomic stability. Certain epigenetic marks in chromatinassociated proteins facilitate DNA double strand break (DSB) repair pathways. Indeed, a wide range of DNA-repair proteins, including PCNA, Rad4, and FANCD2, are modified by ubiquitin to function in translesion synthesis (TLS), change in DNA binding affinity, and recognition of damage lesions, respectively (1). In addition, DNA DSBs cause PTEN dimethyla-

*Corresponding author. Tel: +82-2-820-5242; Fax: +82-2-822-4039; E-mail: sangbs@cau.ac.kr

${ }^{\text {"}}$ These authors contributed equally to this work.

https://doi.org/10.5483/BMBRep.2020.53.2.264

Received 30 October 2019, Revised 18 November 2019, Accepted 15 December 2019

Keywords: Homologous recombination, Methylation dependent protein interaction, PARP1, UHRF1 tion by NSD2 to enhance the PTEN recruitment to DNAdamage sites, which contributes to efficient DNA DSB repair (2).

Ubiquitin-like with PHD and RING finger domain 1 (UHRF1) is involved in DNA methylation regulation via recruitment of DNA methyltransferase 1 (DNMT1) to replication forks (3). Previous studies have shown that UV-induced DNA damage resulted in UHRF1 ubiquitination and proteasomal degradation and that its phosphorylation is related to the destabilization of UHRF1 followed by genomic instability $(4,5)$. Methylation of UHRF1 mediated by SET7, a methyltransferase, showed enhancement of the homologous recombination (HR) pathway through PCNA polyubiquitination (6).

Poly [ADP-ribose] polymerase 1 (PARP1) is recruited to DNA lesions and contributes to DNA repair pathways and genomic stability. Its poly(ADP)ribosylation (PARylation) activity is required for DNA repair and unperturbed replication fork progression (7). Particularly, PARP1 controls HR by recruiting diverse proteins in DNA repair machinery. For example, PARP1 is crucial for the recruitment of breast cancer type 1 susceptibility protein (BRCA1) to DSBs $(8,9)$.

A previous study demonstrated that PARP1 associates with UHRF1 to affect the biological events regulated by UHRF1. Loss of PARP1 proteins led to disruption of the interaction between UHRF1 and DNMT1 and derepression of transcription in heterochromatic domains (10). However, it has remained elusive for additional mechanisms in which both UHRF1 and PARP1 cooperatively promote HR. In this study, we determined the interacting partners of methylated UHRF1 in DNA damage response (DDR) pathways and detected its methylation-dependent binding protein, PARP1. Our results suggest that increased PARP1-UHRF1 interaction correlates with increased cellular functions of $\mathrm{HR}$ and cell cycle progression by contributing to DSB repair choice and to UHRF1-mediated recruitment of DNA repair machinery.

\section{RESULTS}

\section{UHRF1 interacts with PARP1 in methylation-dependent} manner

Previously, we reported the UHRF1 methylation on K385 by methyltransferase SET7, which is a prerequisite for the DSB repair mechanism, HR (6). To determine the regulatory mechanism beyond UHRF1 methylation-dependent HR pro- 
gression, we identified UHRF1-interacting proteins using mass spectrometry proteomic analysis. Two different antibodies against unmethylated or methylated UHRF1 were used to precipitate UHRF1 methylation-dependent interacting proteins. Interestingly, methylated UHRF1 showed a strong interaction with PARP1 (Fig. 1A). After demonstrating that SET7 is responsible for UHRF1 methylation and its catalytically deficient mutant, SET7 H297A, failed to fully methylate UHRF1 (Supplementary Fig. 1), we observed UHRF1 methylation by SET7 is critical for its binding to PARP1 (Fig. 1B). Since we previously demonstrated LSD1 demethylates UHRF1, we hypothesized LSD1 impedes the UHRF1-PARP1 interaction and showed LSD1 inhibition increased their interaction (Fig. 1C). To confirm the correlation between methylation status and UHRF1-PARP1 binding, we analyzed the proximity of these proteins following LSD1 depletion. LSD1 knockdown also induced stronger interaction between UHRF1 and PARP1 (Fig. 1D). Taken together, our data suggest UHRF1 methylation status regulated by SET7 and LSD1 decides the UHRF1-PARP1 interaction.

\section{$\mathrm{H}_{2} \mathrm{O}_{2}$-mediated UHRF1 methylation enhances interaction between UHRF1 and PARP1}

DSBs promote UHRF1 methylation (6). Since we observed UHRF1 interacts with PARP1 in a methylation-dependent manner, we speculated whether DNA damage increases
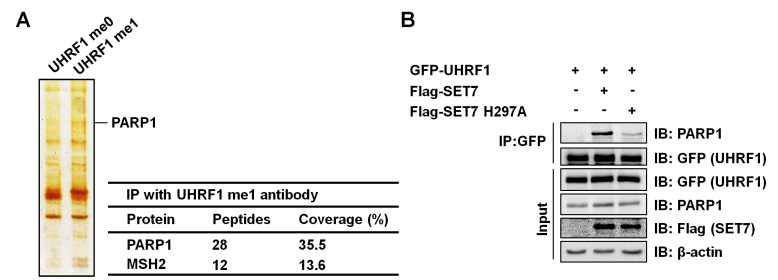

C

D
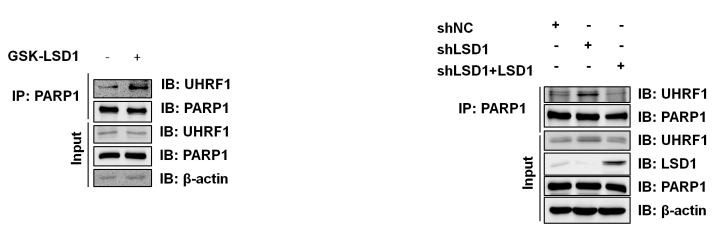

Fig. 1. Methylation status of UHRF1 regulates its interaction with PARP1. (A) Immunoprecipitation (IP) assay using anti-UHRF1 me0 or anti-UHRF1 me1 antibody. The IP complexes were purified from HEK 293T nuclear extracts and visualized using silver staining. Proteins identified by mass spectrometry were indicated. (B) GFP-UHRF1 along with Flag-SET7 WT or catalytically deficient Flag-SET7 H297A were transfected into HCT116 cells, and IPs using anti-GFP antibody were performed. (C) HCT116 cells were treated with $500 \mathrm{nM}$ GSK-LSD1 for $24 \mathrm{~h}$. IPs using anti-PARP1 antibody were performed. (D) Empty vector or Flag-LSD1 was transfected into control cells or stable LSD1 knockdown cells. The lysates were immunoprecipitated using anti-PARP1 antibody.
UHRF1 binding to PARP1. We found the interaction between two proteins was enhanced after $\mathrm{H}_{2} \mathrm{O}_{2}$ exposure. When SET7 was depleted, $\mathrm{H}_{2} \mathrm{O}_{2}$ could not increase UHRF1-PARP1 interaction (Fig. 2A). Next, we showed DNA damage by $\mathrm{H}_{2} \mathrm{O}_{2}$ induces a higher UHRF1-PARP1 interaction in wildtype UHRF1 than in methylation-deficient UHRF1 K385R (Fig. 2B). Together, we demonstrated UHRF1 methylation induced by $\mathrm{H}_{2} \mathrm{O}_{2}$ mediates its binding with PARP1.

\section{PARP1 is required for recruitment of methylated UHRF1 to DNA damage site and $H R$ progression}

The UHRF1 recruitment to damaged lesion is regulated by its methylation status (6). Additionally, we showed PARP1 preferentially interacts with methylated UHRF1 (Fig. 1, 2). Since PARP1 is involved in various DNA repair pathways and functions as a mediator of damage repair proteins $(11,12)$, we asked whether PARP1 recruits UHRF1 to DSB lesion. To measure the UHRF1 recruitment around DSBs, we performed ChIP assay in U2OS-DRGFP cells (Fig. 3A). We generated DSBs by ectopically expressing I-Scel and observed the UHRF1 recruitment. Surprisingly, UHRF1 failed to accumulate to DSBs following PARP1 depletion (Fig. 3B). To investigate the involvement of PARP1 in recruiting UHRF1 to lesion, we used PARP1 inhibitor, olaparib. Consistent with the previous studies that PARP1 is stalled to damaged lesion with olaparib (13), UHRF1 was also stalled around DSBs (Fig. 3C). However, interestingly, we showed UHRF1 K385R could not be recruited to damaged lesion, supporting methylation-dependent UHRF1-PARP1 interaction (Fig. 3D).

As significant role of PARP1 in HR has emerged (14), we hypothesized accumulation of methylated UHRF1 by PARP1 could promote HR. In cells with UHRF1 wildtype, PARP1 knockdown lowered HR efficiency. Interestingly, when the K385 of UHRF1 is disrupted, PARP1 depletion did not affect HR efficiency (Fig. 3E). Likewise, methylation-deficient UHRF1-overexpressing cells showed defective HR both in the presence or absence of olaparib (Fig. 3F). Overall, our data
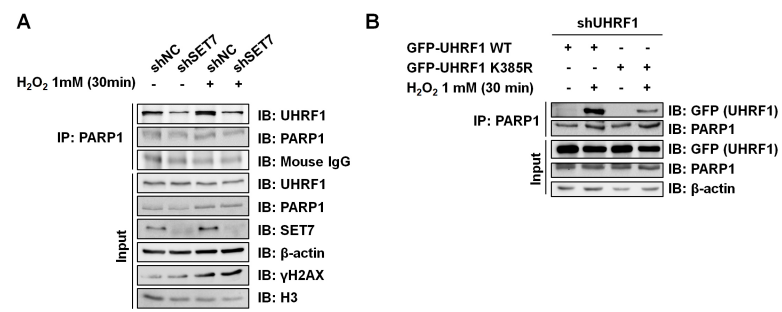

Fig. 2. Damage-induced methylation of UHRF1 enhances interaction between UHRF1 and PARP1. (A) Control cells and SET7 knockdown stable cells treated with $1 \mathrm{mM} \mathrm{H}_{2} \mathrm{O}_{2}$ for 30 min were immunoprecipitated using anti-PARP1 antibody. (B) UHRF1 knockdown stable cells overexpressed with GFP-UHRF1 WT or K385R were treated with $1 \mathrm{mM} \mathrm{H}_{2} \mathrm{O}_{2}$ for $30 \mathrm{~min}$. The lysates were immunoprecipitated using anti-PARP1 antibody. 
A

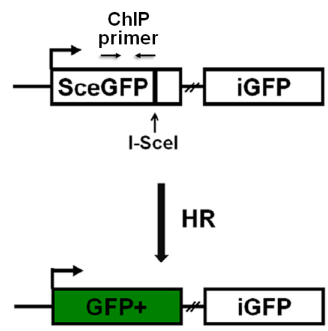

D

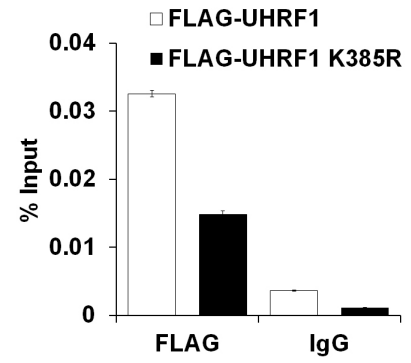

B

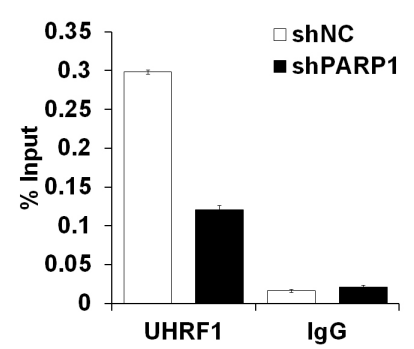

E

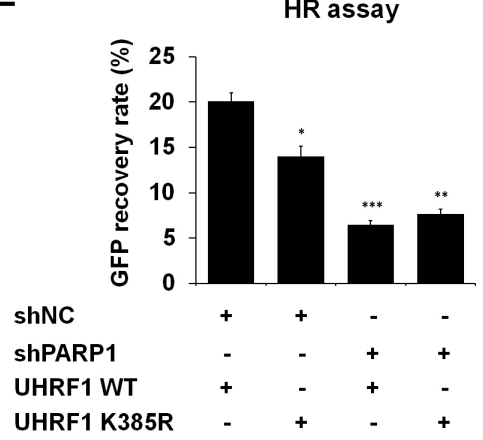

C

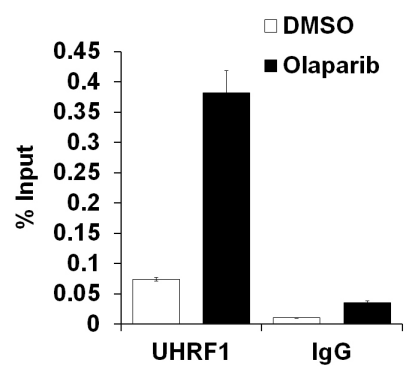

$\mathbf{F}$

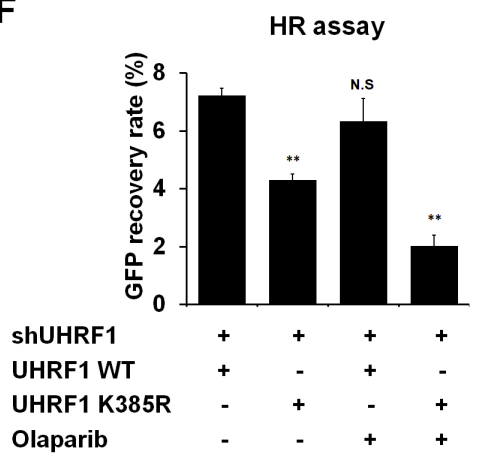

Fig. 3. PARP1 is essential for recruitment of UHRF1 to DNA damage site and HR progression. (A) Schematic representation of a primer pair for the chromatin immunoprecipitation (ChIP) assay in HR reporter with the GFP gene. (B) U2OS-DRGFP cells were transiently knocked down by shNC or shPARP1, transfected with I-Scel $24 \mathrm{~h}$ later, and cells were cross-linked for the ChIP assay $48 \mathrm{~h}$ later. The ChIP-qPCR analyses were performed using anti-UHRF1 antibody on I-Scel DSB site. \% Input indicates UHRF1-bound chromatin fraction relative to input material (total chromatin). (C) Cells were transfected with I-Scel, treated with DMSO or olaparib $24 \mathrm{~h}$ later, and cells were cross-linked for the ChIP assay $48 \mathrm{~h}$ later. The ChIP-qPCR analyses were performed using anti-UHRF1 antibody on I-Scel DSB site. (D) Stable UHRF1 knockdown U2OS-DRGFP cells were transfected with Flag-tagged UHRF1 WT or K385R in addition to I-Scel, treated with olaparib $24 \mathrm{~h}$ later, and cells were cross-linked for the ChIP assay $48 \mathrm{~h}$ later. The ChIP-qPCR analyses were performed using anti-Flag antibody on I-Scel DSB site. Values represent the mean \pm SD of technical duplicates from a representative experiment. All experiments were performed in triplicate with similar results. (E) U2OS cells integrated with HR reporter were transiently knocked down by shNC or shPARP1 and transfected with UHRF1 WT or K385R for measuring HR efficiency. Results were shown as the mean \pm SEM, $\mathrm{n}=3 ; * \mathrm{P}<0.05, * * \mathrm{P}<0.01, * * * \mathrm{P}<0.001$. (F) Cells were transiently knocked down with shUHRF1 and transfected with UHRF1 WT or K385R for measuring HR efficiency. HR reporter assays were performed following $10 \mu \mathrm{M}$ olaparib treatment for $24 \mathrm{~h}$. Results were shown as the mean \pm SEM, $\mathrm{n}=3 ; * * \mathrm{P}<0.01$, N.S: no significant difference.

suggested the role of PARP1 in UHRF1 recruitment to damaged lesion for HR progression.

\section{Interaction of UHRF1-PARP1 regulates cell cycle progression and cell proliferation in response to DNA damage}

Since PARP1-UHRF1 interaction compromised HR activity, we tested whether cooperation of UHRF1 methylation and PARP1 affects cell cycle progression. Consistent with the previous studies $(15,16)$, we observed $\mathrm{H}_{2} \mathrm{O}_{2}$-induced DNA damage increased G2/M-phase proportion in cells with wildtype UHRF1. However, UHRF1 K385R caused significant G2/M-phase arrest, indicating less effective DNA repair (34.6\% WT versus $41.4 \%$ K385R). In addition, olaparib treatment similarly contributed to cell cycle disturbance after recovery of UHRF1 WT or K385R, suggesting PARP1 determines
DSB repair function of UHRF1 (Fig. 4A). Consistent with the result from cell cycle analysis by PI staining, BrdU incorporation also showed the inability of methylation-deficient UHRF1 to efficiently repair damaged DNA and implied the role of PARP1 in tethering UHRF1 to chromatin following olaparib treatment (Supplementary Fig. 2). Interestingly, immunocytochemistry demonstrated that ectopic expression of UHRF1 resulted in a loss of $\gamma-\mathrm{H} 2 \mathrm{AX}$ foci by a successful DSB repair, which was in contrast with remaining $\gamma-\mathrm{H} 2 \mathrm{AX}$ foci observed in adjacent nontransfected cells (Supplementary Fig. 3); however, overexpression of UHRF1 K385R had no detectable effect on $\gamma-\mathrm{H} 2 \mathrm{AX}$ levels, suggesting a failure to overcome the block of cell cycle progression due to the absence of downstream DNA repair processes (Supplementary Fig. 3). Next, we observed the methylation-deficient UHRF1 induced higher apoptotic 
A

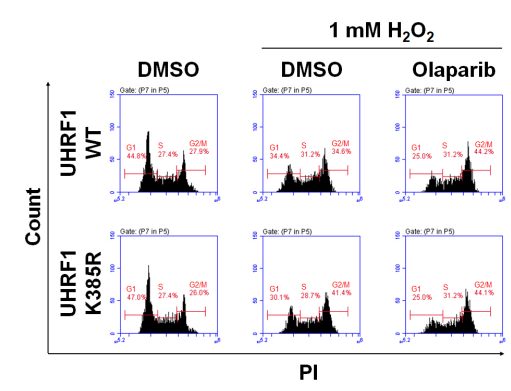

C

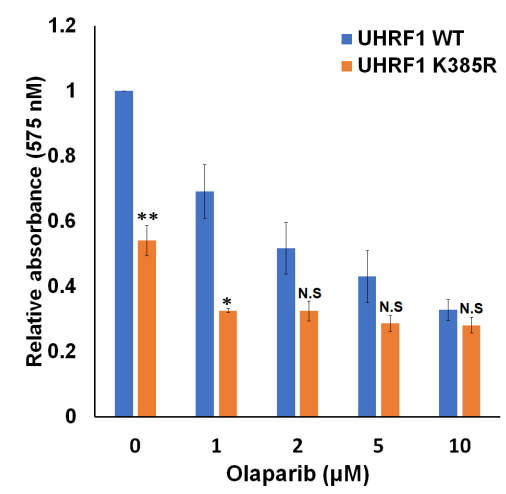

B
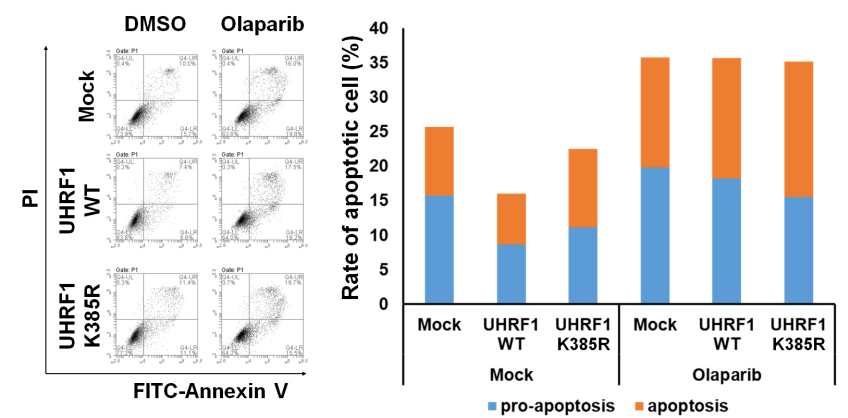

D
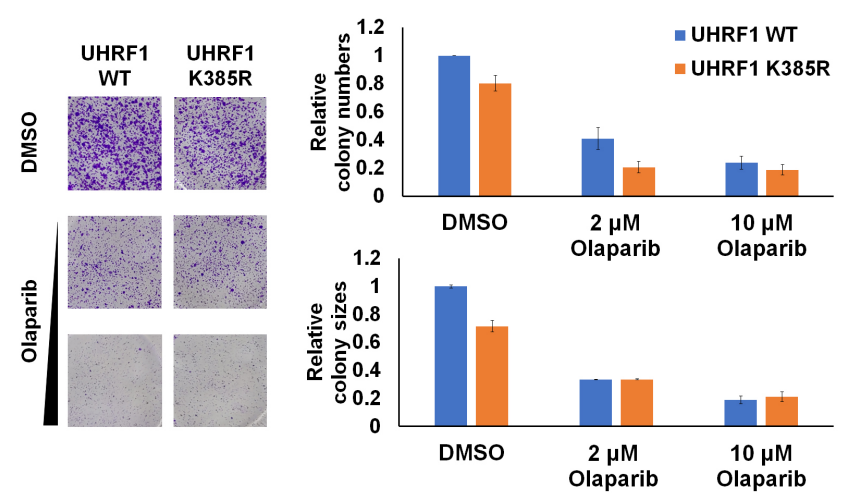

Fig. 4. PARP1 and UHRF1 cooperate for cell cycle progression and cell proliferation in response to DNA damage. (A) Stable UHRF1 knockdown HCT116 cells transfected with UHRF1 WT or K385R were treated with DMSO or olaparib for 24 h. Following 1 mM $\mathrm{H}_{2} \mathrm{O}_{2}$ treatment for $30 \mathrm{~min}$, cells were incubated in fresh media for $24 \mathrm{~h}$, fixed, stained with propidium iodide (PI), and the DNA content was measured by fluorescence-activated cell sorting (FACS). (B) Cells were transfected with UHRF1 WT or K385R and treated with $10 \mu \mathrm{M}$ olaparib for $24 \mathrm{~h}$. FACS analyses of apoptotic cells using Annexin-V and PI staining, showing the \% of apoptotic cells (Annexin- $\mathrm{V}$ positive + Annexin-V and PI double positive). (C) Cell viability was determined using the MTT assay. Cells were transfected with UHRF1 WT or $\mathrm{K} 385 \mathrm{R}$ and treated with various concentrations of olaparib for $24 \mathrm{~h}$. Afterwards, the cells were treated with $1 \mathrm{mM} \mathrm{H}_{2} \mathrm{O}_{2}$ for $30 \mathrm{~min}$ and incubated in fresh media with olaparib for 4 days. Results were shown as the mean \pm SEM, $n=3 ; * P<0.05, * * P<0.01$, N.S: no significant difference. (D) Representative colony formation assay using UHRF1 knockdown HCT116 cells transfected with UHRF1 WT or K385R. Cells were treated with olaparib for 24 h. Afterward, the cells were treated with $100 \mu M \mathrm{H}_{2} \mathrm{O}_{2}$ for 4 days. Values represent the mean \pm SD of technical duplicates from a representative experiment. All experiments were performed in triplicate with similar results.

cell death than that by wildtype UHRF1. However, after olaparib treatment, apoptotic pathway were not suppressed despite overexpression of either wildtype or methylationdeficient UHRF1 (Fig. 4B). Finally, we analyzed the cell viability following $\mathrm{H}_{2} \mathrm{O}_{2}$ treatment in the presence or absence of olaparib. Olaparib significantly decreased cell viability in the cells with wildtype UHRF1. However, regardless of olaparib treatment, UHRF1 K385R did not cause reduced cell viability, indicating higher sensitivity to DNA damage (Fig. 4C). To validate the effect of UHRF1-PARP1 on damage tolerance, we performed the colony formation assay. Consistent with the result described in Fig. 4C, cells with methylationdeficient UHRF1 had little effect on cell viability when treated with olaparib (Fig. 4D). Together, our data showed UHRF1-
PARP1 interaction is critical for cell proliferation.

Overall, our results indicate PARP1 regulates UHRF1 recruitment by its methylation status and the interaction between UHRF1 and PARP1 mediates HR progression.

\section{DISCUSSION}

Although UHRF1 plays important roles in the DNA repair pathway by serving as an adapter protein of DNA repair proteins, the mechanism of these interactions has not been investigated in detail. In the previous study, we found UHRF1 is methylated at $\mathrm{K} 385$ following DNA DSBs induced by $\mathrm{H}_{2} \mathrm{O}_{2}$ (6). Therefore, it was noteworthy to discover novel binding partners of a methylated UHRF1 in response to DNA damage. 
We identified methylated UHRF1-interacting proteins, including PARP1. As a crucial DDR factor, PARP1 mediates DSB repair pathways of both HR and non-homologous end joining (NHE)). Our study revealed methylated UHRF1 is recruited to DNA damage sites in a PARP1-dependent manner to contribute to the DNA repair pathway. This indicates the early stage of DDR is initiated by PARylation and the interaction between PARP1 and UHRF1 proceeds with the later stage of this DSB repair process for HR function (17).

Together with our previous study (6), this data gives insight into the underlying mechanism in defective DSB repair under conditions of SET7 depletion. We suggested unmethylated UHRF1 failed to function in the HR system owing to defective binding to PARP1 as well as inhibited PCNA polyubiquitination. Moreover, following the studies of the antagonistic methylation regulation by SET7 and $\operatorname{LSD} 1(6,17,18)$, we investigated whether LSD1-mediated UHRF1 demethylation could play a role in preventing the interaction between PARP1 and UHRF1. Notably, we showed LSD1 knockdown or specific inhibition using GSK-LSD1 facilitated the association of PARP1 and UHRF1. Concomitant high levels of UHRF1 methylation following LSD1 depletion reiterated the enhancement of its binding to PARP1 caused by SET7 overexpression. Therefore, our results imply both SET7 and LSD1 could affect PARP1mediated chromatin recruitment of UHRF1 by altering the methylation status of UHRF1.

When considering other interaction partners of PARP1, PARP1 is necessary for directing BRCA1 to damaged DNA sites $(8,9)$. In addition, a previous study showed BRCA1 is required for UHRF1 recruitment to DSBs to initiate HR (19). Given that UHRF1 phosphorylation is a prerequisite for its binding to BRCA1 and that UHRF1 is methylated only in the phosphorylated form, we suggest the mechanism of PARP1-dependent UHRF1 recruitment could be mediated by BRCA1. Further studies are necessary to decipher the order of sequential events, including UHRF1 recognition by BRCA1 and UHRF1 methylation by SET7.

Although we did not examine the MSH2, a DNA mismatch repair (MMR) protein, identified in our LC-MS/MS as another candidate protein associated with methylated UHRF1, it is necessary to test whether UHRF1 could bind $\mathrm{MSH} 2$ and to analyze the potential functions of their interaction to induce the DNA repair pathway. Since it is responsible for MMR processes and HR repair (20), we hypothesize UHRF1 might modulate MSH2-mediated DNA repair efficiency.

Overall, our study demonstrated the role of UHRF1 methylation and subsequent interaction with PARP1 in the regulation of DDR through HR promotion and cell proliferation. We provided a new perspective of UHRF1 localization at DSB sites by linking PARP1 binding to UHRF1 chromatin recruitment. However, future experiments are necessary to reveal the precise regions of UHRF1 that are responsible for its positioning on the PARP1 protein surface. The key function of UHRF1 is to maintain DNA methylation; however, it is possible that UHRF1 serves as a regulator of both faithful DNA replication and efficient DNA repair, depending on the biological role of interacting partners following induction of a specific chromatin environment. Therefore, it was fitting to search for a novel UHRF1-binding protein along with different stress signals, including various types of DNA damage, in order to understand the mechanism that causes genome instability.

\section{MATERIALS AND METHODS}

\section{Cell culture and reagents}

293T and U2OS cells were grown in DMEM medium (Gibco), while HCT116 cells were grown in RPMI 1640 medium (Gibco) containing 10\% fetal bovine serum (Gibco) and 0.05\% penicillin-streptomycin (Welgene) at $37^{\circ} \mathrm{C}$ in $5 \% \mathrm{CO}_{2}$.

\section{Immunoprecipitation assay}

Cells were lysed in lysis buffer (50 mM Tris-HCl [pH 7.5], 200 $\mathrm{mM} \mathrm{NaCl}, 0.5 \% \mathrm{NP}-40,1 \times$ protease inhibitor cocktail) and incubated with indicated antibodies overnight at $4^{\circ} \mathrm{C}$. Protein A/G agarose beads (GenDEPOT) were then added, and the mixture was rotated for $3 \mathrm{~h}$ at $4^{\circ} \mathrm{C}$. Bound proteins were analyzed by immunoblotting.

\section{Chromatin immunoprecipitation}

U2OS-DRGFP cells integrated the I-Scel site in chromosomes were used as described previously (21). Briefly, cells were transfected with I-Scel plasmids that induce DSBs and cross-linked with $1 \%$ formaldehyde $48 \mathrm{~h}$ after transfection. This was followed by the addition of $125 \mathrm{mM}$ glycine for 5 min. Harvested cells were resuspended in SDS lysis buffer [1\% SDS, $10 \mathrm{mM}$ EDTA, $50 \mathrm{mM}$ Tris- $\mathrm{HCl}$ (pH 8.1)]. Cells were then sonicated, and the lysates were subjected to immunoprecipitation. The immunoprecipitates were eluted and reverse cross-linked, after which the DNA fragments were purified. The UHRF1-associated DNA was analyzed by real-time PCR using the primer at the I-Scel site. The primer sequence was as follows: forward, 5'-AACCATGTTCATGCCTTCTT-3'; reverse, 5'-CCTCGTGGGTCTT-CTACTTT-3'. All experiments were performed in triplicate giving similar results.

\section{LTQ-orbitrap mass spectrometry}

IP complexes were resolved by SDS-PAGE and stained with Coomassie brilliant blue. After overnight trypsin digestion at $37^{\circ} \mathrm{C}$, the eluted peptides were separated using a C18 column with a linear gradient $\left(\mathrm{A}: 100 \% \mathrm{H}_{2} \mathrm{O}, 0.1 \%\right.$ formic acid, and $\mathrm{B}$ : $100 \% \mathrm{ACN}$ ) at a flow rate of $300 \mathrm{nl} / \mathrm{min}$. Typically, $2 \mu \mathrm{l}$ of sample was injected. Mass spectrometry was performed using a dual-mass spectrometer (LTQ Orbitrap Velos; Thermo Scientific) coupled to a nano-LC system (EASY nLC; Thermo Scientific). This method consisted of a cycle combining one full MS scan (mass range: 150-2000 m/z). Proteins were identified by searching the MS/MS spectra using SEQUEST. 
Mass spectrometry and proteomic analyses were carried out using Ion Mobility Tandem Mass Spectrometer at the Korea Basic Science Institute.

\section{DNA repair assay}

Integrated DNA repair reporter systems were used to determine HR efficiency. Briefly, $24 \mathrm{~h}$ after infection of shUHRF1 or shPARP1 virus, U2OS cells were transfected with I-Scel plasmids, which induce DSBs. Cells were harvested $48 \mathrm{~h}$ after transfection and the percentage of GFP-positive cells were analyzed by fluorescence-activated cell sorting (FACS) using a BD Accuri C6 cytometer (BD Biosciences). Data were analyzed using BD Accuri C6 software (BD Biosciences). Repair frequencies are the mean of at least three independent experiments.

\section{ACKNOWLEDGEMENTS}

This work was supported by Basic Science Research program through the National Research Foundation of Korea Grant (NRF) funded by the Ministry of Science and Technology (NRF-2017R1A2B4004407); and Grant from the NRF funded by the Ministry of Science, Information and Communication Technology (ICT), and Future Planning (NRF-2019R1A4A200 1609).

\section{CONFLICTS OF INTEREST}

The authors have no conflicting interests.

\section{REFERENCES}

1. Bergink $S$ and Jentsch S (2009) Principles of ubiquitin and SUMO modifications in DNA repair. Nature 458, 461-467

2. Zhang J, Lee YR, Dang F et al (2019) PTEN Methylation by NSD2 Controls Cellular Sensitivity to DNA Damage. Cancer Discov 9, 1306-1323

3. Liu X, Gao Q, Li P et al (2013) UHRF1 targets DNMT1 for DNA methylation through cooperative binding of hemi-methylated DNA and methylated H3K9. Nat Commun 4, 1563

4. Chen $\mathrm{H}, \mathrm{Ma} \mathrm{H}$, Inuzuka $\mathrm{H}$ et al (2013) DNA damage regulates UHRF1 stability via the $\mathrm{SCF}($ beta-TrCP) E3 ligase. Mol Cell Biol 33, 1139-1148

5. Yang J, Liu K, Yang J et al (2017) PIM1 induces cellular senescence through phosphorylation of UHRF1 at Ser311. Oncogene 36, 4828-4842

6. Hahm JY, Kim JY, Park JW et al (2019) Methylation of UHRF1 by SET7 is essential for DNA double-strand break repair. Nucleic Acids Res 47, 184-196
7. Ray Chaudhuri A and Nussenzweig A (2017) The multifaceted roles of PARP1 in DNA repair and chromatin remodelling. Nat Rev Mol Cell Biol 18, 610-621

8. Bunting SF, Callen E, Wong N et al (2010) 53BP1 inhibits homologous recombination in Brca1-deficient cells by blocking resection of DNA breaks. Cell 141, 243-254

9. Li M and Yu X (2013) Function of BRCA1 in the DNA damage response is mediated by ADP-ribosylation. Cancer Cell 23, 693-704

10. De Vos M, El Ramy R, Quenet D et al (2014) Poly(ADP-ribose) polymerase 1 (PARP1) associates with E3 ubiquitin-protein ligase UHRF1 and modulates UHRF1 biological functions. J Biol Chem 289, 16223-16238

11. Young LM, Marzio A, Perez-Duran P et al (2015) TIMELESS Forms a Complex with PARP1 Distinct from Its Complex with TIPIN and Plays a Role in the DNA Damage Response. Cell Rep 13, 451-459

12. Jelinic P and Levine DA (2014) New insights into PARP inhibitors' effect on cell cycle and homology-directed DNA damage repair. Mol Cancer Ther 13, 1645-1654

13. Clements $\mathrm{KE}$, Thakar $\mathrm{T}$, Nicolae $\mathrm{CM}$, Liang $\mathrm{X}$, Wang HG and Moldovan GL (2018) Loss of E2F7 confers resistance to poly-ADP-ribose polymerase (PARP) inhibitors in BRCA2-deficient cells. Nucleic Acids Res 46, 8898-8907

14. Rimar KJ, Tran PT, Matulewicz RS, Hussain M and Meeks JJ (2017) The emerging role of homologous recombination repair and PARP inhibitors in genitourinary malignancies. Cancer 123, 1912-1924

15. Thorn T, Gniadecki R, Petersen AB, Vicanova J and Wulf HC (2001) Differences in activation of G2/M checkpoint in keratinocytes after genotoxic stress induced by hydrogen peroxide and ultraviolet $\mathrm{A}$ radiation. Free Radic Res 35, 405-416

16. Santa-Gonzalez GA, Gomez-Molina A, Arcos-Burgos M, Meyer JN and Camargo M (2016) Distinctive adaptive response to repeated exposure to hydrogen peroxide associated with upregulation of DNA repair genes and cell cycle arrest. Redox Biol 9, 124-133

17. Liu C, Vyas A, Kassab MA, Singh AK and Yu X (2017) The role of poly ADP-ribosylation in the first wave of DNA damage response. Nucleic Acids Res 45, 8129-8141

18. Wang J, Hevi S, Kurash JK et al (2009) The lysine demethylase LSD1 (KDM1) is required for maintenance of global DNA methylation. Nat Genet 41, 125-129

19. Zhang $\mathrm{H}$, Liu $\mathrm{H}$, Chen $\mathrm{Y}$ et al (2016) A cell cycledependent BRCA1-UHRF1 cascade regulates DNA doublestrand break repair pathway choice. Nat Commun 7, 10201

20. Yang Q, Zhang R, Wang XW et al (2004) The mismatch DNA repair heterodimer, $\mathrm{hMSH} / 6$, regulates BLM helicase. Oncogene 23, 3749-3756

21. Chen G, Chen J, Qiao Y et al (2018) ZNF830 mediates cancer chemoresistance through promoting homologousrecombination repair. Nucleic Acids Res 46, 1266-1279 